\title{
Influence of optical feedback on laser frequency spectrum and threshold conditions
}

\author{
Osmundsen, Jens Henrik; Gade, Niels
}

Published in:

I E E E Journal of Quantum Electronics

Link to article, DOI:

10.1109/JQE.1983.1071857

Publication date:

1983

Document Version

Publisher's PDF, also known as Version of record

Link back to DTU Orbit

Citation (APA):

Osmundsen, J. H., \& Gade, N. (1983). Influence of optical feedback on laser frequency spectrum and threshold conditions. I E E E Journal of Quantum Electronics, 19(3), 465-469. https://doi.org/10.1109/JQE.1983.1071857

\section{General rights}

Copyright and moral rights for the publications made accessible in the public portal are retained by the authors and/or other copyright owners and it is a condition of accessing publications that users recognise and abide by the legal requirements associated with these rights.

- Users may download and print one copy of any publication from the public portal for the purpose of private study or research.

- You may not further distribute the material or use it for any profit-making activity or commercial gain

- You may freely distribute the URL identifying the publication in the public portal

If you believe that this document breaches copyright please contact us providing details, and we will remove access to the work immediately and investigate your claim 
coated with positive-type photoresist (AZ-1350) and exposed to mercury light for 1 minute after prebaking at $90^{\circ} \mathrm{C}$ for 20 minutes. Then, the lasers were operated at $20^{\circ} \mathrm{C}, 5 \mathrm{~mW} /$ facet for 20 minutes. After this, they were syringed in the acetone solution, with ultrasonic vibration, for 3 minutes. The photoresists coated on three lasers were completely dissolved, but in two lasers, no matter what, only part of the photoresist coated at the emitting area could not be dissolved. Note that remaining photoresist was observed on either, but not both, mirrors of the laser. The actinic absorbance of the photoresist decays suddenly in light with a wavelength above $0.5 \mu \mathrm{m}$, so $0.8 \mu \mathrm{m}$ radiation from a laser is transparent to the photoresist. We have certified that photoresist baked at $150^{\circ} \mathrm{C}$ for 20 minutes could be dissolved completely in the acetone solution, but not $180^{\circ} \mathrm{C}$ for 20 minutes. Thus, we concluded that a temperature rise of about $150^{\circ} \mathrm{C}$ did take place at the mirror surface at least in two lasers. Similar results have been presented by $O$. Matsuda et al. at the 26 th Nat. Conv. Japan. Soc. Appl. Phys., Spring 1979 (in Japanese).

[22] Y. Nannichi, J. Matsui, and K. Ishida, "Rapid degradation in double-heterostructure lasers-II: Semiquantitative analyses on the propagation of dark line defects," Japan. J. Appl. Phys., vol. 14, pp. 1561-1568, Oct. 1975.
[23] T. Kobayashi, T. Kawakami, and Y. Furukawa, "Thermal diagnosis of dark lines in degraded GaAs-AlGaAs double-heterostructure lasers," Japan. J. Appl. Phys., vol. 14, pp. 508-515, Apr. 1975.



Ken'ich Mizuishi was born in Chiba, Japan, on April 1, 1948. He received the B.S. degree in electrical engineering from the Waseda University, Tokyo, Japan, in 1971.

In 1971 he joined the Central Research Laboratory, Hitachi Ltd., Tokyo, Japan. From 1971 to 1975 he was engaged in the research and development of millimeter-wave GaAs SBD's and IMPATT diodes, and from 1975 to 1977 he was involved in investigating the reliability of low-noise GaAs MESFET's. He is currently engaged in the reliability research of semiconductor lasers including 0.7-0.8 $\mu \mathrm{m}(\mathrm{Al}, \mathrm{Ga}) \mathrm{As} / \mathrm{GaAs}$ lasers and $1.3 \mu \mathrm{m} \mathrm{InGaAsP} / \mathrm{InP}$ lasers.

Mr. Mizuishi is a member of the Japan Society of Applied Physics and the Institute of Electronics and Communication Engineers of Japan.

\title{
Influence of Optical Feedback on Laser Frequency Spectrum and Threshold Conditions
}

\author{
JENS HENRIK OSMUNDSEN AND NIELS GADE
}

\begin{abstract}
The steady state behavior of the external cavity operated laser has been analyzed, taking into account multiple reflections.

The effect of optical feedback is included in the phase- and gainconditions by a factor which is shown to have a simple geometrical representation. From this representation it is easily seen how the laser frequency spectrum and the threshold gain depend on external parameters such as distance to the reflection point and the amount of optical feedback.

Furthermore, by inserting a variable attenuator in the external cavity and measuring the threshold current versus transmittance we have simultaneously determined the photon lifetime and the absolute amount of optical feedback. For the laser considered we found the photon lifetime $\tau_{p}=1.55 \mathrm{ps}$.
\end{abstract}

Manuscript received October 4, 1982.

The authors are with the Electromagnetics Institute, Technical University of Denmark, Lyngby, Denmark.

\section{INTRODUCTION}

T is well known that external optical feedback strongly affects the properties of semiconductor lasers. The reflected light causes variations in, e.g., the threshold gain, output power, and output spectrum [1], [2].

Most of the models dealing with these problems neglect multiple reflections in the external cavity and simply incorporate the optical feedback by adding a time delayed feedback term to the standard laser equation [3], [4] .

The purpose of the present work has been to analyze the influence of optical feedback on the laser frequency spectrum and on the threshold gain, taking into account multiple reflections.

A complex feedback parameter is introduced and the phase 
and gain conditions are derived in Section II-A. Two cases appear to be qualitatively different, namely the cases where the external reflection coefficient is smaller than or larger than the reflection coefficient of the laser mirror facing the external cavity. These cases are treated in Section II-B and II-C, respectively. The influence of optical feedback on the threshold current is analyzed in Section II-D. Finally in Section III, we describe the experimental determination of the photon lifetime.

\section{THEORY}

\section{A. Basic Equations}

A model for the external cavity operated laser is shown in Fig. 1. Here $r_{2}$ and $r_{3}$ are amplitude reflection coefficients and $d$ and $L$ are the lengths of the laser diode and the external cavity, respectively.

In order to account for multiple reflections in the external cavity the boundary value problem for the electrical fields in the compound cavity has to be solved. This has been done in [1], where the threshold condition for laser oscillations is given by the secular equation

$$
e^{(g-\alpha) d}=\frac{1+r_{2} r_{3} e^{-j \phi_{o}}}{r_{2} e^{-j \phi_{1}}\left[r_{2}+r_{3} e^{-j \phi_{o}}\right]}
$$

where the parameters are



and $\phi_{0}=\omega \tau$, where $\tau$ is the round trip time in the external cavity and $\omega$ is the angular frequency of the laser light. Furthermore, $\phi_{1}=\left(\omega / \omega_{D}\right) 2 \pi$, where $\omega_{D}=2 \pi f_{d}=\pi c_{g} / d$ is the angular frequency mode spacing and $c_{g}$ is the group velocity.

We introduce the complex feedback parameter

$$
z=\frac{1+r_{2} r_{3} e^{-j \phi_{o}}}{1+\left(r_{3} / r_{2}\right) e^{-j \phi_{o}}}=e^{G+j \phi}
$$

where $G=\ln |z|$ and $\phi=\operatorname{Arg} z$.

In the following we shall for simplicity assume $r_{3}$ to be real. The case of complex $r_{3}$ could be dealt with by including the phase in $\phi_{o}$. The threshold condition (1) can then be written

$$
e^{(g-\alpha) d}=r_{2}^{-2} e^{j \phi_{1}} z \text {. }
$$

$z$ describes the change in phase and threshold gain due to optical feedback. The effective reflection coefficient of the FabryPerot cavity defined by the laser mirror and the external cavity can then be written as [5]

$$
r_{\mathrm{eff}}=r_{2} z^{-1} \text {. }
$$

From (3) the phase and gain conditions become

$$
\begin{aligned}
\phi_{1} & =p \cdot 2 \pi-\phi & \text { (phase condition) } \\
g & =\alpha+\frac{1}{d} \ln \frac{1}{r_{2}^{2}}+\frac{1}{d} G & \text { (gain condition) }
\end{aligned}
$$

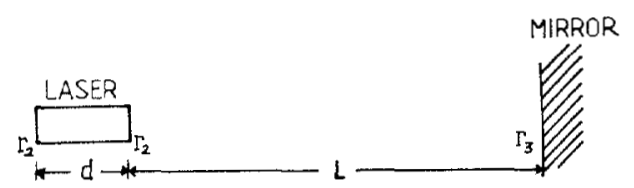

Fig. 1. Schematic representation of the external cavity operated laser.

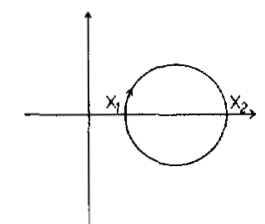

(a)

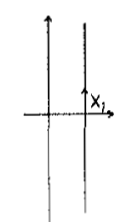

(b)

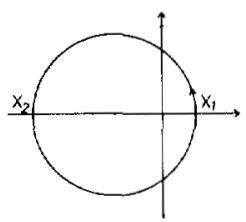

(c)
Fig. 2. Loci of $z\left(\phi_{o}\right)$ in the complex plane. (a) $r_{3}<r_{2}$, (b) $r_{3}=r_{2}$, (c) $r_{3}>r_{2}$. Arrows indicate direction of revolution for increasing $\phi_{0}$.

where $p$ is an integer. In the case of no optical feedback $\left(r_{3}=0\right),(5)$ reduce to the well-known expressions [1]

$$
\begin{aligned}
\phi_{1} & =p \cdot 2 \pi \Longleftrightarrow \omega=p \cdot \omega_{D} \\
g & =\alpha+\frac{1}{d} \ln \frac{1}{r_{2}^{2}} .
\end{aligned}
$$

In the phase condition (5a) the additional term $\phi$ accounts for the influence of optical feedback, while the term $(1 / d) G$ in the gain condition $(5 b)$ represents the excess required gain for the laser to oscillate.

The parameter $z$ is a periodic function of $\phi_{o}$ with period $2 \pi$. The locus of $z\left(\phi_{o}\right)$ as $\phi_{o}$ varies is a circle in the complex plane (see Fig. 2) with center

$$
\left(x_{o}, y_{o}\right)=\left(\frac{r_{2}^{2}\left(1-r_{3}^{2}\right)}{r_{2}^{2}-r_{3}^{2}}, 0\right)
$$

and radius

$$
R=\frac{r_{2} r_{3}\left(1-r_{2}^{2}\right)}{\left|r_{2}^{2}-r_{3}^{2}\right|} \text {. }
$$

The intersections with the $x$ axis are

$$
\begin{aligned}
& x_{1}=\frac{r_{2}\left(1+r_{2} r_{3}\right)}{r_{2}+r_{3}} \text { for } \phi_{o}=0(\bmod 2 \pi) \\
& x_{2}=\frac{r_{2}\left(1-r_{2} r_{3}\right)}{r_{2}-r_{3}} \text { for } \phi_{o}=\pi(\bmod 2 \pi) .
\end{aligned}
$$

The required excess gain $G$ due to optical feedback lies between $G_{\min }=\ln x_{1}$ and $G_{\max }=\ln \left|x_{2}\right|$, depending on the phase of the reflected field.

We have $G=G_{\min }$ for $\phi_{o}=0(\bmod 2 \pi)$, i.e., when the reflected field is in phase with the emitted field. It is seen from Fig. 3 that $G_{\min }$ decreases monotonically for $r_{3}$ increasing from 0 to 1 .

For the reflected field in counter phase with the emitted field, $\phi_{o}=\pi(\bmod 2 \pi)$, we have $G=G_{\max }$. For $r_{3}$ approaching $r_{2}, G_{\max }$ increases to infinity. This is to be expected since the effective reflection coefficient $r_{\text {eff }}$ equals 0 for $r_{2}=r_{3}$ and $\phi_{o}=\pi(\bmod 2 \pi)$, i.e., the external cavity acts as a FabryPerot cavity with 100 percent transmission. 


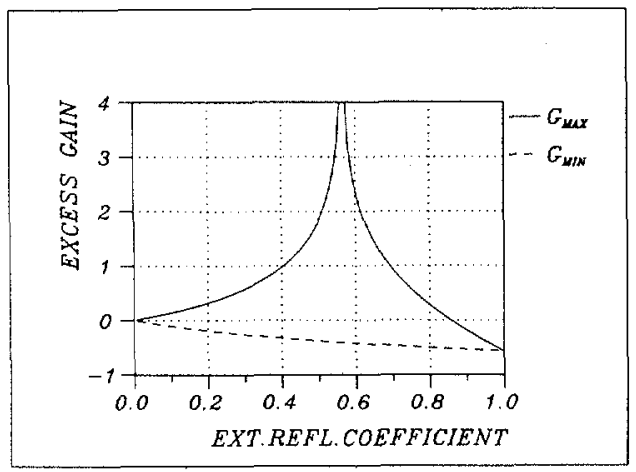

Fig. 3. Upper and lower bounds on $G$ as a function of the optical feedback.

\section{PHASE CONDITION}

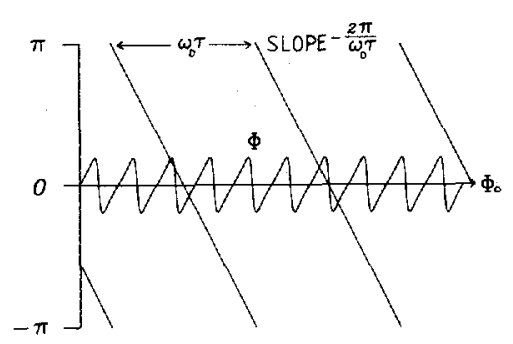

Fig. 4. Phase condition for $r_{3}=0.4$.

For $r_{3}=1$ the threshold gain becomes $g=\alpha+1 / d \ln 1 / r_{2}$ in both cases $\phi_{o}=0(\bmod 2 \pi)$ and $\phi_{o}=\pi(\bmod 2 \pi)$. This is to be expected since $\left|r_{\mathrm{eff}}\right|=1$, independently of the reflectivity of the mirror facing the external cavity.

\section{B. Small Reflections $\left(r_{3}<r_{2}\right)$}

We now consider the phase condition in the case $r_{3}<r_{2}$. Using $\phi_{1}=\left(\omega / \omega_{D}\right) 2 \pi(5 a)$ can be written

$$
\phi=-\frac{\omega}{\omega_{D}} 2 \pi+p \cdot 2 \pi=-\frac{2 \pi}{\omega_{D} \tau} \phi_{O}+p \cdot 2 \pi
$$

The graphical interpretation of (9) is shown in Fig. 4.

It is seen that solutions to the phase condition will occur in groups centered around the angular frequencies given by

$$
\frac{2 \pi}{\omega_{D} \tau} \phi_{O}=p \cdot 2 \pi \Longleftrightarrow \omega=p \omega_{D}
$$

i.e., the longitudinal frequencies of the laser without external cavity. The frequency spacing between the solutions within each longitudinal mode group will be slightly less than $f=1 / 2 \tau$.

The argument $\phi$ varies periodically between $\pm \phi_{\max }$, where $\phi_{\max }$ is given by (see Fig. 2)

$$
\sin \phi_{\max }=\frac{R}{x_{o}}=\frac{r_{3}\left(1-r_{2}^{2}\right)}{r_{2}\left(1-r_{3}^{2}\right)} \text {. }
$$

$\phi_{\max }$ is a measure of the maximum achievable frequency shift. From (9) this maximum frequency shift is

$$
\Delta \omega_{\max }=\frac{\phi_{\max }}{2 \pi} \omega_{D}
$$

It is seen from Fig. 2 that $\phi_{\max }<\pi / 2$ for $r_{3}<r_{2}$. This means

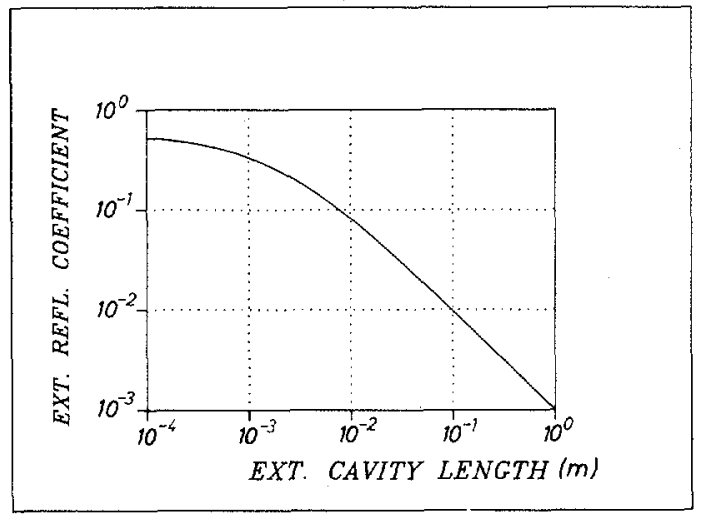

Fig. 5. Condition for single mode operation within each longitudinal mode group.

that solutions will only occur in the intervals

$$
I_{p}=\left[p \omega_{D}-\frac{\omega_{D}}{4}, p \omega_{D}+\frac{\omega_{D}}{4}\right] .
$$

From Fig. 4 it is easily seen how the solutions to the phase condition are affected by the amount of optical feedback. Reducing $r_{3}$ causes a decrease of $\phi_{\max }$, which again causes a reduction of the number of solutions to the phase condition.

The distance to the external reflection point also affects the number of solutions. For decreasing distance the slope of the straight lines becomes steeper (more negative), causing a decrease in the number of solutions.

In general, the phase condition has multiple solutions, but for certain combinations of the external cavity length $L$ and the external reflection coefficient $r_{3}$, there is only one solution to the phase condition present within each interval $I_{p}$, i.e., each mode group consists of only one mode. This is ensured if the slope $-2 \pi / \omega_{D} \tau$ of the straight line exceeds the minimum slope of the $\phi$-curve.

The maximum value $r_{\max }$, for which there is only one solution in $I_{p}$, is given by (see Fig. 5)

$r_{\max }=\frac{1+r_{2}^{2}+f_{d} \tau\left(1-r_{2}^{2}\right)-\sqrt{\left(1+r_{2}^{2}+f_{d} \tau\left(1-r_{2}^{2}\right)\right)^{2}-4 r_{2}^{2}}}{2 r_{2}}$.

As long as $r_{\max } \ll 1$, the values determined by (14) are in good agreement with [2], in which a first order model is used, i.e., multiple reflections have been neglected.

In Fig. 6 is shown the variation of $\phi$ and $G$ versus $\phi_{o}$. From this figure and Fig. 4 it is seen that only solutions corresponding to intersection points near $\phi_{o}=0(\bmod 2 \pi)$ will have lowthreshold gains. Therefore, each longitudinal mode group in the laser emission spectrum will consist of a number of modes separated by $f \lesssim 1 / \tau$ and with increasing intensity as the wavelength corresponding to a longitudinal mode of the free running laser is approached (see Fig. 7).

The allowed frequencies have to satisfy the analytical expression

$$
p \omega_{D}-\omega=\frac{\omega_{D}}{2 \pi} \operatorname{Arctan}\left[\frac{r_{3}\left(1-r_{2}^{2}\right) \sin \omega \tau}{r_{2}\left(1+r_{3}^{2}\right)+r_{3}\left(1+r_{2}^{2}\right) \cos \omega \tau}\right] .
$$






Fig. 6. Variation of $G=\ln |z|$ and phase $\phi$ of the complex feedback parameter $z\left(r_{3}=0.4\right)$.

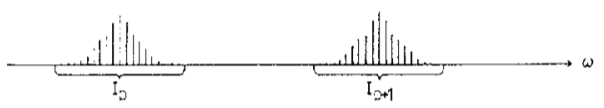

Fig. 7. Laser emission spectrum consisting of longitudinal mode groups. The modes within each group are separated by $f \leqslant 1 / \tau$. There are no solutions to the phase condition in the intermediate interval.

For small reflections $\left(r_{3} \ll 1\right)$ the first order approximation becomes

$$
p \omega_{D}-\omega=\frac{\omega_{D}}{2 \pi} \frac{r_{3}\left(1-r_{2}^{2}\right)}{r_{2}} \sin \omega \tau
$$

This is the same expression for the frequency shift caused by external reflections as given in [3] and [4], where first order models have been used. This approximation is only good in the case of small reflections. For large amounts of optical feedback, multiple reflections cannot be neglected, and (5a) has to be used in determining the allowed frequencies.

\section{Large Reflections $\left(r_{3}>r_{2}\right)$}

In the case $r_{3}>r_{2}$, the argument $\phi$ is no longer restricted to the interval $-\pi / 2<\phi<\pi / 2$, but will vary in the whole range $-\pi<\phi \leqslant \pi$. Solutions to the phase condition will thus occur all over the frequency axis.

In Fig. 8 is shown the variation of the argument $\phi$ and the excess required gain $G$ versus $\phi_{o}$.

The phase condition is fulfilled for every period of $\phi_{o}$, but only solutions corresponding to intersection points near the $\phi_{o}$ axis, i.e., angular frequencies near $p \cdot \omega_{D}$, have low-threshold gains. Therefore, the laser emission spectrum will consist of longitudinal mode groups which no longer are clearly separated. The mode spacing is $f \lesssim 1 / \tau$, and as in the previous case, the intensity of the modes increases as the wavelength corresponding to a longitudinal mode of the free running laser is approached (Fig. 9).

\section{Threshold Current Reduction}

The threshold gains are calculated from (5b) for the frequencies satisfying (5a). The onset of laser oscillations will take place at the frequency $\omega_{o}$ with lowest threshold gain.

Assuming a linear relationship between gain and current

$g=\beta I$



Fig. 8. Variation of $G=\ln |z|$ and the phase $\phi$ of the complex feedback parameter $z\left(r_{3}=0.6\right)$.

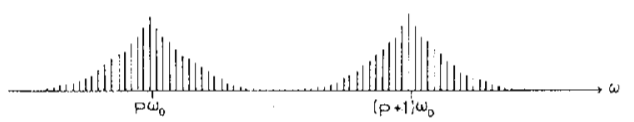

Fig. 9. Laser emission spectrum for $r_{3}>r_{2}$. The solutions to the phase condition occur all over the frequency axis with spacing $f \lesssim 1 / \tau$.

leads to the following expression for the threshold current.

$$
I_{\text {th }}=I_{o}\left\{1+f_{d} \tau_{p} \ln \left[r_{2}^{2} \frac{1+r_{2} r_{3}\left(r_{2} r_{3}+2 \cos \omega_{o} \tau\right)}{r_{2}^{2}+r_{3}^{2}+2 r_{2} r_{3} \cos \omega_{o} \tau}\right]\right\}
$$

where $I_{o}$ is the threshold current for the free running laser and $\tau_{p}$ is the photon lifetime,

$$
\tau_{p}=\frac{1}{\beta I_{o} c_{g}}
$$

In the first order approximation $\left(r_{3} \ll 1\right)(18)$ reduces to

$$
I_{t h}=I_{o}\left(1-2 f_{d} \tau_{p} \frac{r_{3}\left(1-r_{2}^{2}\right)}{r_{2}} \cos \omega_{o} \tau\right)
$$

which is consistent with earlier derived expressions based on models neglecting multiple reflections [4].

Measuring $I_{t h}$ versus the external reflection coefficient makes it possible to determine the photon lifetime $\tau_{p}$.

\section{EXPERIMENTAL}

In order to measure the threshold current versus the amount of optical feedback, we inserted a variable attenuator in the external cavity. The variable attenuator was of type NRC 925 B, and the laser diode used was a Hitachi CSP-laser.

The threshold current was measured versus transmittance $T$ of the variable attenuator. The corresponding reflection coefficient therefore becomes $r_{3}=k T$ where $k$ is a coupling parameter including external mirror reflectivity and coupling between laser and external cavity.

We used a large external cavity length $(31 \mathrm{~cm})$ and could therefore assume the laser to oscillate at $\phi_{o}=0(\bmod 2 \pi)$. In this case (18) becomes

$$
I_{t h}=I_{o}\left(1+2 f_{d} \tau_{p} \ln \left[r_{2} \frac{1+r_{2} k T}{r_{2}+k T}\right]\right)
$$




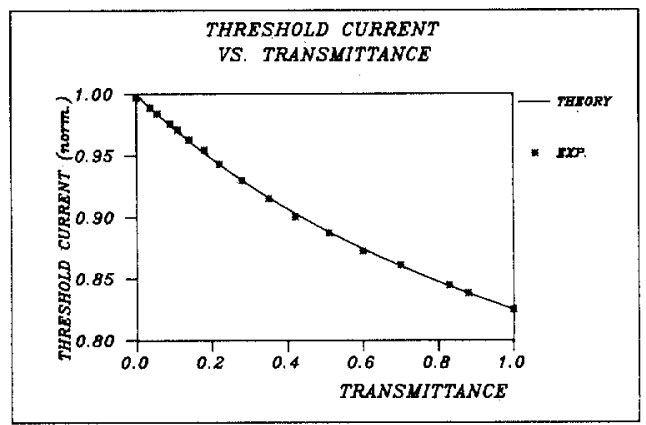

Fig. 10. Normalized threshold current versus transmittance of variable attenuator. The solid curve is calculated from (21) using the optimized values of $\tau_{p}$ and $k$.

Using the measured value of the mode spacing $f_{d}=125 \mathrm{GHz}$ the best fit to the experimental points was found for $\tau_{p}=1.55$ ps and $k=0.65$ (see Fig. 10). Within an approximate 90 percent confidence region $\tau_{p}$ was determined to $\tau_{p}=1.55 \pm 0.15 \mathrm{ps}$.

It should be noticed that the threshold current reduction is proportional to the product $\tau_{p} \cdot k$ to first order in $r_{3}$. This means that in order to determine $\tau_{p}$ and $k$ separately, the amount of optical feedback should be as large as possible. Therefore, a better coupling between laser and external cavity will lead to a better determination of $\tau_{p}$.

Using the measured value of the diode cavity length $d=320$ $\mu \mathrm{m}$, the value of $\tau_{p}$ corresponds to an absorption coefficient $\alpha=45 \mathrm{~cm}^{-1}$. This result is consistent with $\alpha$-values used elsewhere [6].

\section{CONCLUSION}

We have analyzed the steady state behavior of the external cavity operated laser taking into account multiple reflections. In this analysis a complex feedback parameter has been introduced.

From the geometrical representation of this parameter it has been shown how external optical feedback influences the laser frequency spectrum and threshold gain. A condition for single mode operation within each longitudinal mode group has been given. Also, analytical expressions have been given for frequency shift and threshold current reduction due to optical feedback, and it has been shown that these expressions in the first order approximation reduce to earlier derived expressions based on models neglecting multiple reflections.

Furthermore, by measuring the threshold current versus the transmittance of a variable attenuator in the external cavity, the photon lifetime and the absolute amount of optical feedback have simultaneously been determined. The result of the measurement is in good agreement with expectations, and it shows that this method is a very convenient way of determining the photon lifetime $\tau_{p}$ without making any assumption on the coupling efficiency between laser and external cavity.

\section{ACKNOWLEDGMENT}

The authors would like to thank B. Tromborg, Electromagnetics Institute, for many useful suggestions and discussions. Also, $\mathrm{H}$. Olesen is acknowledged for comments on the manuscript.

\section{REFERENCES}

[1] A. Olsson and C. L. Tang, "Coherent optical interference effects in external cavity semiconductor lasers," IEEE J. Quantum Electron., vol. QE-17, pp. 1320-1323, Aug. 1981.

[2] L. Goldberg, H. F. Taylor, A. Dandridge, J. F. Weller, and R. O. Miles, "Spectral characteristics of semiconductor lasers with optical feedback," IEEE Trans. Microwave Theory Tech., vol. MTT-30, pp. 401-410, Apr. 1982.

[3] R. Lang and K. Kobayashi, "External optical feedback effects on semiconductor injection laser properties," IEEE J. Quantum Electron., vol. QE-16, pp. 347-355, Mar. 1980.

[4] O. Hirota and Y. Suematsu, "Noise properties of injection lasers due to reflected waves," IEEE J. Quantum Electron., vol. QE-15, pp. 142-149, Mar. 1979.

[5] H. A. Haus, "Theory of modelocking of a laser diode in an external resonator," J. Appl. Phys., vol. 51, no. 8, pp. 4042-4049, Aug. 1980.

[6] M. W. Fleming and A. Mooradian, "Fundamental line broadening of single-mode (GaAl)As diode lasers," Appl. Phys. Lett., vol..38, p. $511,1981$.

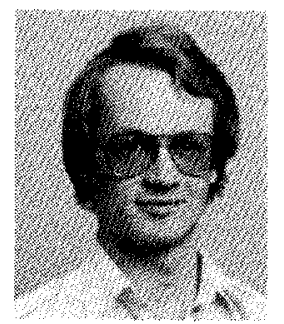

Jens Henrik Osmundsen was born on August 8, 1958. He received the M.Sc. degree in electrical engineering from the Technical University of Denmark, Lyngby, in 1982.

He is now with the Electromagnetics Institute, Technical University of Denmark, where he is working on properties and applications of external-cavity operated lasers.

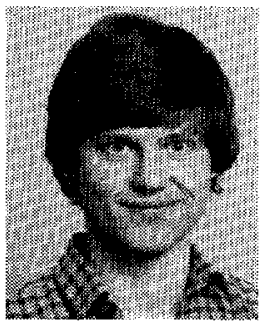

Niels Gade was born on March 14, 1958 in Aarhus, Denmark. He received the M.Sc. degree in electrical engineering in January 1982 from the Technical University of Denmark, Lyngby, Denmark, where he is now working on a Ph.D. degree at the Electromagnetics Institute. His current interests are in the field of semiconductor lasers, in particular ps pulse generated, mode locking, and external cavity operation. 